\title{
Limited value for urinary 5-HIAA excretion as prognostic marker in gastrointestinal neuroendocrine tumours
}

\author{
Wouter T Zandee, Kimberly Kamp, Roxanne C S van Adrichem, Richard A Feelders \\ and Wouter W de Herder
}

Department of Internal Medicine, Sector Endocrinology, ENETS Centre of Excellence, Erasmus MC Cancer Institute, Erasmus MC, Rotterdam, The Netherlands
Correspondence should be addressed to W W de Herder Email

w.w.deherder@erasmusmc.nl

\begin{abstract}
Objective: To determine if urinary 5-hydroxyindoleacetic acid (5-HIAA) excretion is of prognostic value for overall survival (OS) in patients with a gastrointestinal neuroendocrine tumour (NET) and to compare the prognostic value with patient characteristics, ENETS/WHO grading, ENETS TNM staging and biomarkers.

Design and methods: Data was collected from patients with a gastrointestinal NET or a NET with gastrointestinal metastases and available 5-HIAA excretion in 24-h urine samples. Laboratory results were stratified for urinary 5-HIAA and chromogranin A (CgA): $<2 \times$ upper limit of normal (ULN), 2-10x ULN, or $>10 \times$ ULN. For neuron-specific enolase (NSE), this was the reference range or $>1 \times$ ULN. OS was compared using Kaplan-Meier and log-rank tests, and hazard ratios were calculated using Cox regression for univariate and multivariate analyses.

Results: A total of 371 patients were included, $46.6 \%$ female with a mean age of 59.9 years. OS was shortest in patients with urinary 5-HIAA excretion $>10 \times$ ULN vs reference range (median 83 months vs 141 months, $P=0.002$ ). In univariate analysis, urinary 5-HIAA excretion >10x ULN was a negative predictor (HR 1.62, 95\% Cl: 1.09-2.39). However, in multivariate analysis, only age (HR 1.04, 95\% Cl: 1.01-1.08), grade 3 disease (HR 5.09, 95\% Cl: 2.20-11.79), NSE >1× ULN (HR 2.36, 95\% Cl: 1.34-4.14) and CgA >10× ULN (HR 3.61, 95\% Cl: 1.56-8.34) remained as the predictors. Conclusion: Urinary 5-HIAA excretion $>10 \times$ ULN is a negative predictor for OS. However, when added to other biomarkers and grading, it is no longer a predictor for OS. Therefore, it should only be determined to assess carcinoid syndrome and not for prognostic value.
\end{abstract}

\section{Introduction}

Although neuroendocrine tumours (NETs) are rare, they are well known for their production and secretion of polypeptide hormones, which results in distinct clinical syndromes. NETs that originate from the midgut most often produce serotonin, causing not only carcinoid syndrome with flushing and diarrhoea but also carcinoid heart disease, which can result in right-sided heart failure (1). Serotonin levels may vary over the day and therefore its metabolite 5-hydroxyindoleacetic acid (5-HIAA) is usually measured in $24-\mathrm{h}$ urine samples. The urinary
C 2016 European Society of Endocrinology Printed in Great Britain
5-HIAA excretion has been shown to be a valuable tool for the diagnosis of small intestinal NETs (mainly in the presence of liver metastases), and it correlates with severity of heart disease in the carcinoid syndrome $(2,3)$. During the follow-up of patients with midgut NETs, urinary 5-HIAA excretion is usually determined in combination with chromogranin A ( $\mathrm{CgA}$ ) and neuron-specific enolase (NSE) to assess the status of the disease, especially in the so-called functioning (hypersecreting or syndromic) NETs (3). Plasma 5-HIAA may become available in the 
near future, as it seems to be a good predictor of carcinoid heart disease, but at present, it is hardly validated as a prognostic marker in the follow-up of NETs $(4,5,6)$.

$\mathrm{CgA}$ is secreted by most NETs, including gastroenteropancreatic (GEP) NETs, pheochromocytomas and lung carcinoids and both functioning and nonfunctioning NETs (7). It reflects tumour burden, and it has been demonstrated to be an important marker for the diagnosis and prognosis in patients with GEP-NETs (8). In several studies, a high level of serum CgA has been shown to be an unfavourable factor for survival and, therefore, should be routinely measured during follow-up $(9,10,11,12,13,14)$. NSE is the third circulating tumour marker, which can be used in the follow-up of NETs and is usually elevated in patients with poorly differentiated NETs and small-cell lung cancer. For the diagnosis of NETs, serum NSE is less specific, but together with serum $\mathrm{CgA}$, its sensitivity might increase and it can predict survival as well $(15,16)$.

However, discussion remains on the usefulness of the urinary 5-HIAA excretion (and maybe plasma 5-HIAA levels) as a prognostic factor for survival in GEP-NET and lung NET patients, as it seems to be inferior to serum chromogranin A and only a few publications report on its prognostic value (3). Elevated urinary 5-HIAA excretion was a negative predictor of survival in a study by Janson et al., but was no longer a predictor when compared with serum CgA (17). Additional studies confirmed the predictive value of the urinary 5-HIAA excretion, but it was not compared with serum $\mathrm{CgA}$ and serum NSE, as especially serum $\mathrm{CgA}$ is nowadays routinely used in the follow-up of NETs $(18,19,20,21,22)$. In this retrospective study, our aim was to determine once more if the urinary 5-HIAA excretion is a prognostic marker for overall survival (OS) in patients with gastrointestinal NETs and to compare it with serum CgA and serum NSE in combination with ENETS TNM staging, ENETS/WHO grading and other patient or tumour characteristics as nowadays they are routinely used in clinical practice.

\section{Methods}

\section{Patients}

All records of patients who were treated for a NET between 1993 and 2012 in the ENETS Centre of Excellence for Neuroendocrine Tumours, Erasmus MC, Rotterdam, The Netherlands were analysed. Patients were included if they had a gastrointestinal NET with liver metastases and at least one 24-h urine sample of 5-HIAA was available at the time of diagnosis or referral to our centre. Patients with liver metastases from unknown primary origins were also included when they presented with a mesenteric lymph node deposit typical for midgut tumours or if (functional) imaging showed no evidence of a lung, pancreas, kidney or ovarium NET. Patients diagnosed with the multiple endocrine neoplasia (MEN) type1 and von Hippel-Lindau (VHL) disease were excluded.

Patient characteristics, pathology data, laboratory results and imaging findings were all recorded. Furthermore, data on treatment modalities during follow-up were recorded and date of last visit or, if applicable, date of death. NETs were diagnosed on the basis of a combination of markers, imaging and histology (including synaptophysin and chromogranin staining) according to the current guidelines $(9,23)$.

\section{Tumour markers}

5-HIAA was determined in 24-h urine samples and measured using the reverse-phase high-performance liquid chromatography (HPLC) with fluorimetric detection. The reference range is below $50 \mu \mathrm{mol} / 24 \mathrm{~h} \mathrm{(24)}$. If available, two 24-h samples were used and the average was calculated, but if only one sample was available this was reported.

CgA in serum was measured using a solid-phase, twosite IRMA assay (Cisbio Bioassays) with an upper limit of normal (ULN) of $94 \mu \mathrm{g} / \mathrm{L}$. NSE in serum was measured using an electrochemiluminescence immunoassay on an immunoassay analyzer (Roche Diagnostics) and has an ULN of $16.2 \mu \mathrm{g} / \mathrm{L}$.

Results were stratified for levels of 24-h urinary 5-HIAA excretion, serum CgA and serum NSE. Because slight elevation of 24-h urinary 5-HIAA excretion and serum $\mathrm{CgA}$ is often due to interference or dietary incompliance, reference range was defined as values being below $2 \times$ ULN. Furthermore, urinary 5-HIAA excretion and serum CgA were stratified as, high (2-10x ULN) and very high ( $>10 \times$ ULN).

Patients with a 5-HIAA excretion below $10 \mu \mathrm{mol} / 24 \mathrm{~h}$ were excluded because of probable inappropriate 24-h sampling.

Serum NSE was stratified for being either high (above ULN) or normal (within reference range).

\section{Statistical analysis}

All markers were recorded at diagnosis or referral to our centre, to determine the prognostic value for survival. 
Primary outcome was overall survival (OS) and hazard ratios of possible predictors of survival in univariate and multivariate analyses.

Differences between groups on baseline were tested with chi-square for categorical data and with an one-way ANOVA for continuous data. OS was analysed using the Kaplan-Meier method and log-rank test to determine the significant differences between the mortality in the groups, according to the range of 5-HIAA. Hazard ratios were calculated using a Cox-regression analysis. Both univariate and multivariate analyses were performed. A $P$ value of $<0.05$ was considered statistically significant.

Calculations were performed using SPSS for Windows software (version 23.0, SPSS).

\section{Results}

A total of 374 patients were identified with a gastrointestinal NET or NET from unknown origin, with liver metastases and available 24-h 5-HIAA urine samples. Three patients were excluded as they had 5-HIAAs below $10 \mu \mathrm{mol} / \mathrm{L}$ leaving 371 patients available for analyses. Of these patients, $84.6 \%$ had provided two urine samples at the first visit. All patients were included in the baseline and univariate analyses, but 171 patients had one or more missing values and were excluded from the multivariate analysis.

Baseline clinical characteristics are shown in Table 1. Patients had an average age of $59.9 \pm 10.5$ years and $46.6 \%$ were female. Patients mostly presented with a small intestinal NET or only with a mesenteric node metastasis and with grade 1 or 2 tumours. Forty-seven patients (12.7\%) presented with liver metastases with unknown primary tumour. At referral, 136 (36.7\%) patients were already using a somatostatin analogue. Unfortunately, data on tumour grade were only available for $59.5 \%$ of patients.

Patients with very high 24-h urinary 5-HIAA excretion had the highest incidence of somatostatin analogue use $(P=0.002)$. They also had the highest serum NSE and CgA levels, but this was not significant (Table 2). During
Table 1 Overall baseline characteristics $(n=371)$.

\begin{tabular}{lr}
\hline Female, $n(\%)$ & $173(46.6)$ \\
Age (years \pm SD) & $59.9 \pm 10.5$ \\
Primary tumour, $n(\%)$ & $195(52.5)$ \\
Small intestine & $34(9.2)$ \\
Ileocoecal & $36(9.7)$ \\
Colon & $6(1.6)$ \\
Appendix & $53(14.3)$ \\
Mesenterial node & $47(12.7)$ \\
Unknown & \\
Grade, $n(\%)$ & $108(29.1)$ \\
Grade 1 (Ki67 $\leq 2 \%)$ & $98(26.4)$ \\
Grade 2 (Ki67 3-20\%) & $11(3.0)$ \\
Grade 3 (Ki67>20\%) & $154(41.5)$ \\
Missing & $136(36.6)$ \\
Use of somatostatin analogue, $n(\%)$ &
\end{tabular}

follow-up, 27 patients (23.9\%) who had a normal 24-h urinary 5-HIAA excretion at baseline developed elevated 5-HIAA excretion, mainly between 2-10x ULN.

\section{Overall survival}

Overall, 159 patients died during a median follow-up of 115 months. When stratified for the 24-h urinary 5-HIAA excretion, OS was shortest in patients with a very high 24-h urinary 5-HIAA excretion and longest in patients with normal 5-HIAA excretion (Fig. 1 and Table 3, $P=0.03$ ). Median survival varied from 141 months in the group with normal 24-h urinary 5-HIAA excretion to 83 months in the group with very high 24-h urinary 5-HIAA excretion, corresponding to five-year survivals of respectively, 74.0 and $63.0 \%$.

\section{Prognostic factors}

In univariate analysis, very high 24-h urinary 5-HIAA excretion was associated with a shorter survival compared with the reference range with hazard ratios of 1.09 (95\% CI: 0.73-1.63) for high 5-HIAA and 1.62 (95\% CI: 1.09-2.39) for very high 5-HIAA (Table 4). However, in a multivariate analysis 24 -h urinary 5-HIAA excretion is no longer a prognostic marker in this

Table 2 Baseline characteristics of groups based on 5-HIAA values. Numerical data are presented as mean \pm s.D. Differences were tested with $\chi^{2}$ for categorical data and with ANOVA for numerical data.

\begin{tabular}{lc} 
& Reference range $(n=165)$ \\
\cline { 1 - 2 } Age & $58.3 \pm 11.7$ \\
NSA & $5210.8 \pm 34109$ \\
Use of SSA & $28.0 \pm 87.8$ \\
& $28(24.8 \%)$ \\
\hline
\end{tabular}

\begin{tabular}{c}
$\mathbf{2 - 1 0 \times} \mathbf{U L N}(n=159)$ \\
\hline $60.9 \pm 10.6$ \\
$2321.7 \pm 13784$ \\
$19.5 \pm 37.6$ \\
$54(37.2 \%)$
\end{tabular}

\begin{tabular}{|c|c|}
\hline$>\mathbf{1 0} \times \mathbf{U L N}(n=119)$ & $P$ value \\
\hline $60.4 \pm 8.9$ & 0.11 \\
\hline $7778.4 \pm 18096$ & 0.17 \\
\hline $40.3 \pm 117.0$ & 0.19 \\
\hline $54(47.8 \%)$ & 0.002 \\
\hline
\end{tabular}




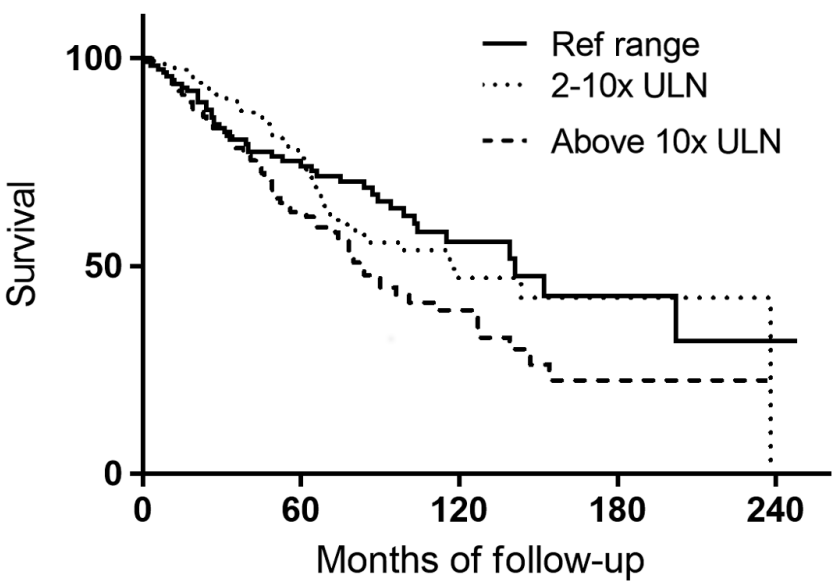

\section{Figure 1}

Survival stratified for urinary 5-HIAA excretion.

population, with hazard ratios of 0.76 (95\% CI: $0.45-1.88)$ and 0.92 (95\% CI: 056-1.61) for high and very high 5-HIAA (Table 5). Sex was also not associated with survival. However, age at diagnosis, serum CgA and NSE were negative predictors of survival. Furthermore, grade 3 tumours were associated with the highest hazard ratio of 5.09 (95\% CI: 2.20-11.79). Adding baseline use of a somatostatin analogue to the multivariate analysis did not significantly change the model.

\section{Discussion}

Current ENETS guidelines are inconclusive on the value of the 24-h urinary 5-HIAA excretion to determine the prognosis in patients with GEP-NETs (3). Although serum CgA and NSE are widely reported as predictors for survival, few studies show any additional value for urinary 5-HIAA excretion $(17,18,19,20,21,22)$. The 24-h urinary 5-HIAA excretion was studied as a predictor for survival in our study. We were able to predict survival stratified for urinary 5-HIAA excretion with the Kaplan-Meier method and very high 24-h urinary 5-HIAA excretion was identified as a negative predictor for survival in patients with a midgut NET. However, in multivariate analysis, other tumour markers (serum CgA and serum NSE) and tumour grade were far more powerful predictors and the 24-h urinary 5-HIAA excretion was no longer a significant predictor in this multivariate analysis. Therefore, 24-h urinary 5-HIAA excretion seems to have no additional value for predicting prognosis in follow-up when serum CgA and serum NSE are already used.

A limited number of other studies in GEP-NET patients have shown that elevated 24-h urinary 5-HIAA excretion is associated with a shorter survival, but these studies did not compare the urinary 5-HIAA excretion with serum CgA or NSE $(18,19,20,21,22)$. Only Janson et al. have compared urinary 5-HIAA excretion with serum CgA in GEP-NET patients using a multivariate analysis and demonstrated, as in our study, that urinary 5-HIAA excretion had no additional value for the prognosis of patients with GEP-NETs when other contemporary biomarkers are used (17). Additionally, we confirmed these results using serum NSE, in combination with staging and grading.

On the other hand, the use of 24-h urinary 5-HIAA excretion in the follow-up of GEP-NET patients cannot be completely abolished. The determination of plasma or 24-h urinary 5-HIAA excretion has been shown to be positively correlated with the severity of the carcinoid syndrome $(6,25)$. However, simply obtaining the history from patients should suffice during follow-up. Moreover, current clinical trials prefer questionnaires to determine flushing episodes and diarrhoea frequency and volumes over this biochemical marker. Its value, therefore, lies mainly in its predictive value for carcinoid heart disease. In this context, it should still be measured at diagnosis and at follow-up $(2,6,25)$. Current ENETS guidelines recommend annual cardiac screening in patients with the carcinoid syndrome, but in clinical practice there is diversity in local screening protocols for carcinoid heart disease $(9,26)$. It is currently unclear if one urinary sample with non-elevated urinary 5-HIAA excretion values at baseline is sufficient to rule out the development of carcinoid heart disease. In addition, it was not determined how often during follow-up re-evaluation of the urinary 5-HIAA excretion should be undertaken for a timely decision on further cardiac testing. In this respect the measurement of plasma NT-proBNP levels (maybe including serum CgA and

Table 3 Overall survival curves stratified for urinary 5-HIAA excretion. Kaplan-Meier estimate of overall survival for patients with a gastrointestinal NET, stratified for urinary 5-HIAA excretion. Curves were compared with a log-rank test $(P=0.002)$.

\begin{tabular}{|c|c|c|}
\hline Numbers at risk & 0 month & 24 months \\
\hline Ref range & 113 & 100 \\
\hline $2-10 \times$ ULN & 145 & 135 \\
\hline$>10 \times$ ULN & 113 & 96 \\
\hline
\end{tabular}

\begin{tabular}{|c|c|}
\hline 48 months & 72 months \\
\hline 72 & 54 \\
\hline 102 & 52 \\
\hline 69 & 45 \\
\hline
\end{tabular}

\begin{tabular}{c}
96 months \\
\hline 36 \\
32 \\
27
\end{tabular}

\begin{tabular}{c}
120 months \\
\hline 21 \\
20 \\
20
\end{tabular}

\begin{tabular}{c}
144 months \\
\hline 11 \\
9 \\
9
\end{tabular}

$\begin{array}{ccc}\frac{168 \text { months }}{7} & & \text { 240 months } \\ & & 1 \\ 6 & & 0 \\ & 0\end{array}$

www.eje-online.org 
Table 4 Univariate analysis of 5-HIAA.

\begin{tabular}{|c|c|}
\hline & Hazard ratio $(95 \% \mathrm{Cl})$ \\
\hline Reference range $(<100 \mu \mathrm{mol} / 24 \mathrm{~h})$ & 1.0 (reference) \\
\hline $2-10 \times$ ULN $(100-500 \mu \mathrm{mol} / 24 \mathrm{~h})$ & $1.09(0.73-1.63)$ \\
\hline$>10 \times$ ULN $(>500 \mu \mathrm{mol} / 24 \mathrm{~h})$ & $1.62(1.09-2.39)$ \\
\hline
\end{tabular}

ULN, upper limit of normal; 5-HIAA, 5-hydroxyindoleacetic acid.

plasma 5-HIAA) might also be more relevant $(6,27)$. In our study cohort, $23.9 \%$ of patients with normal 5-HIAA at baseline had elevated 5-HIAA at follow-up, showing that one negative screening at baseline is not sufficient to rule out serotonin production at follow-up and therefore minimising the risk of carcinoid heart disease on the basis of 24-h urinary 5-HIAA excretion.

Major limitation of our study is the non-protocolled, retrospective design of this study. Not all patients included had supplied two urine samples; however, in light of recent studies and our own unpublished observations, this is probably not a limitation as even shorter collection periods seem to be reliable $(28,29)$. For the multivariate analysis, a large number of patients had to be excluded, mainly due to missing data on Ki67 staining of tumour samples. This resuts in a selection of more recently diagnosed patients, because the MIB-1 staining of tumour samples was introduced only around 2010. However, as this proved to be one of the most powerful predictors of survival, we decided to accept this.

In conclusion, the determination of 24-h urinary 5-HIAA excretion is inferior to other available serum biomarkers for predicting survival in patients with gastrointestinal NETs. Serum CgA and NSE have a higher predictive value, and there is no need for dietary restriction or 24-h urine collections. Urinary 5-HIAA

Table 5 Multivariate analysis of prognostic factors $(n=200)$.

\begin{tabular}{|c|c|}
\hline & Hazard ratio $(95 \% \mathrm{Cl})$ \\
\hline Female & $0.95(0.56-1.61)$ \\
\hline Age & $1.04(1.01-1.08)$ \\
\hline \multicolumn{2}{|l|}{ Grade } \\
\hline Grade 1 & Reference \\
\hline Grade 2 & $1.40(0.79-3.34)$ \\
\hline Grade 3 & $5.09(2.20-11.79)$ \\
\hline \multicolumn{2}{|l|}{ 5-HIAA } \\
\hline Reference range $(100 \mu \mathrm{mol} / 24 \mathrm{~h})$ & Reference \\
\hline 2-10× ULN (100-500 $\mu \mathrm{mol} / 24 \mathrm{~h})$ & $0.76(0.45-1.88)$ \\
\hline$>10 \times$ ULN $(>500 \mu \mathrm{mol} / 24 \mathrm{~h})$ & $0.92(0.56-1.61)$ \\
\hline \multicolumn{2}{|l|}{ Chromogranin A } \\
\hline Reference range $(<188 \mu \mathrm{g} / \mathrm{L})$ & Reference \\
\hline $2-10 \times$ ULN $(188-940 \mu \mathrm{g} / \mathrm{L})$ & $1.59(0.75-3.34)$ \\
\hline$>10 \times$ ULN $(>940 \mu \mathrm{g} / \mathrm{L})$ & $3.61(1.56-8.34)$ \\
\hline NSE above ULN $(>16.2 \mu \mathrm{g} / \mathrm{L})$ & $2.36(1.34-4.14)$ \\
\hline
\end{tabular}

might still be important for determining the potential risk of development of carcinoid heart disease. However, there might be an important role for NT-proBNP, possibly in combination with plasma 5-HIAA and serum $\mathrm{CgA}$, in predicting carcinoid heart disease. It is still unclear whether a negative screening for urinary 5-HIAA excretion is sufficient or that repeated 5-HIAA is required during follow-up for the early determination of the carcinoid heart disease risk.

\section{Declaration of interest}

W T Z, K K and R C S v A have no disclosures to report. R A F received research grants and speakers fee from Novartis. W W d $\mathrm{H}$ served on advisory boards for and received speakers fees from Ipsen and Novartis.

\section{Funding}

This research did not receive any specific grant from any funding agency in the public, commercial or not-for-profit sector.

\section{References}

1 de Herder WW. Biochemistry of neuroendocrine tumours. Best Practice and Research: Clinical Endocrinology and Metabolism 200721 33-41. (doi:10.1016/j.beem.2006.12.002)

2 Zuetenhorst JM, Bonfrer JM, Korse CM, Bakker R, van Tinteren H $\&$ Taal BG. Carcinoid heart disease: the role of urinary 5-hydroxyindoleacetic acid excretion and plasma levels of atrial natriuretic peptide, transforming growth factor-beta and fibroblast growth factor. Cancer 200397 1609-1615. (doi:10.1002/cncr.11226)

3 O'Toole D, Grossman A, Gross D, Delle Fave G, Barkmanova J, O'Connor J, Pape UF, Plockinger U, Mallorca Consensus Conference Participants \& European Neuroendocrine Tumor Society. ENETS consensus guidelines for the standards of care in neuroendocrine tumors: biochemical markers. Neuroendocrinology 200990 194-202. (doi:10.1159/000225948)

4. Tellez MR, Mamikunian G, O’Dorisio TM, Vinik AI \& Woltering EA. A single fasting plasma 5-HIAA value correlates with 24-hour urinary 5-HIAA values and other biomarkers in midgut neuroendocrine tumors (NETs). Pancreas 201342 405-410. (doi:10.1097/ MPA.0b013e318271c0d5)

5 Adaway JE, Dobson R, Walsh J, Cuthbertson DJ, Monaghan PJ, Trainer PJ, Valle JW \& Keevil BG. Serum and plasma 5-hydroxyindoleacetic acid as an alternative to 24-h urine 5-hydroxyindoleacetic acid measurement. Annals of Clinical Biochemistry 201653 554-560. (doi:10.1177/0004563215613109)

6 Dobson R, Burgess MI, Banks M, Pritchard DM, Vora J, Valle JW, Wong C, Chadwick C, George K, Keevil B et al. The association of a panel of biomarkers with the presence and severity of carcinoid heart disease: a cross-sectional study. PLOS ONE 20138 e73679. (doi:10.1371/journal.pone.0073679)

7 Taupenot L, Harper KL \& O'Connor DT. The chromograninsecretogranin family. New England Journal of Medicine 2003348 1134-1149. (doi:10.1056/NEJMra021405)

8 Modlin IM, Gustafsson BI, Moss SF, Pavel M, Tsolakis AV \& Kidd M. Chromogranin A - biological function and clinical utility in neuro endocrine tumor disease. Annals of Surgical Oncology 201017 2427-2443. (doi:10.1245/s10434-010-1006-3)

9 Pape UF, Perren A, Niederle B, Gross D, Gress T, Costa F, Arnold R, Denecke T, Plockinger U, Salazar R et al. ENETS Consensus guidelines 
for the management of patients with neuroendocrine neoplasms from the jejuno-ileum and the appendix including goblet cell carcinomas. Neuroendocrinology 201295 135-156. (doi:10.1159/000335629)

10 Rossi RE, Garcia-Hernandez J, Meyer T, Thirlwell C, Watkins J, Martin NG, Caplin ME \& Toumpanakis C. Chromogranin A as a predictor of radiological disease progression in neuroendocrine tumours. Annals of Translational Medicine 20153 118. (doi:10.3978/j. issn.2305-5839.2015.04.23)

11 Han X, Zhang C, Tang M, Xu X, Liu L, Ji Y, Pan B \& Lou W. The value of serum chromogranin $\mathrm{A}$ as a predictor of tumor burden, therapeutic response, and nomogram-based survival in well-moderate nonfunctional pancreatic neuroendocrine tumors with liver metastases. European Journal of Gastroenterology \& Hepatology 201527 527-535. (doi:10.1097/meg.0000000000000332)

12 Yao JC, Hassan M, Phan A, Dagohoy C, Leary C, Mares JE, Abdalla EK, Fleming JB, Vauthey JN, Rashid A et al. One hundred years after "carcinoid": epidemiology of and prognostic factors for neuroendocrine tumors in 35,825 cases in the United States. Journal of Clinical Oncology 200826 3063-3072. (doi:10.1200/ JCO.2007.15.4377)

13 Seregni E, Ferrari L, Bajetta E, Martinetti A \& Bombardieri E. Clinical significance of blood chromogranin A measurement in neuroendocrine tumours. Annals of Oncology 200112 (Supplement 2) S69-S72. (doi:10.1093/annonc/12.suppl_2.S69)

14 Arnold R, Wilke A, Rinke A, Mayer C, Kann PH, Klose KJ, Scherag A, Hahmann M, Muller HH \& Barth P. Plasma chromogranin A as marker for survival in patients with metastatic endocrine gastroenteropancreatic tumors. Clinical Gastroenterology and Hepatology 20086 820-827. (doi:10.1016/j.cgh.2008.02.052)

15 Oberg K. Circulating biomarkers in gastroenteropancreatic neuroendocrine tumours. Endocrine-Related Cancer 201118 (Supplement 1) S17-S25. (doi:10.1530/ERC-10-0280)

16 van Adrichem RC, Kamp K, Vandamme T, Peeters M, Feelders RA \& de Herder WW. Serum neuron-specific enolase level is an independent predictor of overall survival in patients with gastroenteropancreatic neuroendocrine tumors. Annals of Oncology 201627 746-747. (doi:10.1093/annonc/mdv626)

17 Janson ET, Holmberg L, Stridsberg M, Eriksson B, Theodorsson E, Wilander E \& Oberg K. Carcinoid tumors: analysis of prognostic factors and survival in 301 patients from a referral center. Annals of Oncology 19978 685-690. (doi:10.1023/A:1008215730767)

18 Turner GB, Johnston BT, McCance DR, McGinty A, Watson RG, Patterson CC \& Ardill JE. Circulating markers of prognosis and response to treatment in patients with midgut carcinoid tumours. Gut 200655 1586-1591. (doi:10.1136/gut.2006.092320)

19 van der Horst-Schrivers AN, Post WJ, Kema IP, Links TP, Willemse PH, Wymenga AN \& de Vries EG. Persistent low urinary excretion of 5-HIAA is a marker for favourable survival during follow-up in patients with disseminated midgut carcinoid tumours. European Journal of Cancer 200743 2651-2657. (doi:10.1016/j.ejca.2007.07.025)

20 Formica V, Wotherspoon A, Cunningham D, Norman AR, Sirohi B, Oates J \& Chong G. The prognostic role of WHO classification, urinary 5-hydroxyindoleacetic acid and liver function tests in metastatic neuroendocrine carcinomas of the gastroenteropancreatic tract. British Journal of Cancer 200796 1178-1182. (doi:10.1038/ sj.bjc.6603699)

21 Agranovich AL, Anderson GH, Manji M, Acker BD, Macdonald WC $\&$ Threlfall WJ. Carcinoid tumour of the gastrointestinal tract: prognostic factors and disease outcome. Journal of Surgical Oncology 199147 45-52. (doi:10.1002/jso.2930470111)

22 Hellman P, Lundstrom T, Ohrvall U, Eriksson B, Skogseid B, Oberg K, Tiensuu Janson E \& Akerstrom G. Effect of surgery on the outcome of midgut carcinoid disease with lymph node and liver metastases. World Journal of Surgery 200226 991-997. (doi:10.1007/s00268-0026630-z)

23 Falconi M, Bartsch DK, Eriksson B, Kloppel G, Lopes JM, O'Connor JM, Salazar R, Taal BG, Vullierme MP, O'Toole D et al. ENETS Consensus Guidelines for the management of patients with digestive neuroendocrine neoplasms of the digestive system: welldifferentiated pancreatic non-functioning tumors. Neuroendocrinology 201295 120-134. (doi:10.1159/000335587)

24 van Haard PM. Chromatography of urinary indole derivatives. Journal of Chromatography 1988429 59-94. (doi:10.1016/S03784347(00)83867-0)

25 Bhattacharyya S, Toumpanakis C, Chilkunda D, Caplin ME \& Davar J. Risk factors for the development and progression of carcinoid heart disease. American Journal of Cardiology 2011107 1221-1226. (doi:10.1016/j.amjcard.2010.12.025)

26 Dobson R, Valle JW, Burgess MI, Poston GJ \& Cuthbertson DJ. Variation in cardiac screening and management of carcinoid heart disease in the UK and Republic of Ireland. Clinical Oncology 201527 741-746. (doi:10.1016/j.clon.2015.06.016)

27 Korse CM, Taal BG, de Groot CA, Bakker RH \& Bonfrer JM. Chromogranin-A and N-terminal pro-brain natriuretic peptide: an excellent pair of biomarkers for diagnostics in patients with neuroendocrine tumor. Journal of Clinical Oncology 200927 4293-4299. (doi:10.1200/JCO.2008.18.7047)

28 Gedde-Dahl M, Thiis-Evensen E, Tjolsen AM, Mordal KS, Vatn M \& Bergestuen DS. Comparison of 24-h and overnight samples of urinary 5-hydroxyindoleacetic acid in patients with intestinal neuroendocrine tumors. Endocrine Connections 20132 50-54. (doi:10.1530/EC-120077)

29 Zuetenhorst JM, Korse CM, Bonfrer JM, Peter E, Lamers CB $\&$ Taal BG. Daily cyclic changes in the urinary excretion of 5-hydroxyindoleacetic acid in patients with carcinoid tumors. Clinical Chemistry 200450 1634-1639. (doi:10.1373/clinchem.2004.032151)

Received 6 May 2016

Revised version received 27 July 2016

Accepted 4 August 2016 